\title{
EL DESPOJO DEL PATRIMONIO GALLEGO. UNA DENUNCIA DE ISAAC DÍAZ PARDO
}

\author{
Jesús Ángel Sánchez García \\ María Luisa Sobrino Manzanares \\ Universidade de Santiago de Compostela
}

Dando continuidad al objetivo de recuperar textos sobre arte gallego inéditos o poco conocidos, en esta ocasión se han seleccionado dos artículos escritos por el multifacético e incansable artista y empresario Isaac Díaz Pardo (Santiago de Compostela, 1920), ambos publicados en la revista Galicia Emigrante. El origen de estos artículos se explica por la coincidencia en 1955 en Buenos Aires, tras la caída de la dictadura de Perón, de un Isaac Díaz Pardo recién llegado de Galicia y de Luis Seoane, pintor y diseñador gráfico exiliado en la Argentina desde la Guerra Civil. Este encuentro —Díaz Pardo va a permanecer en la Argentina hasta 1968, con frecuentes idas y venidas a España-, dio como primer fruto el proyecto de un nuevo tipo de empresa cerámica que reunía las intenciones de Díaz Pardo sobre la imbricación de la industria en el medio geológico, histórico y social, con las ideas que Luis Seoane pretendía para el diseño y la investigación formal: la creación de la fábrica argentina de Magdalena (Buenos Aires).

La estancia en Buenos Aires vino a ampliar y dar un contenido a los procesos industriales que Díaz Pardo tenía en mente, pero sobre todo sirvió para poner en comunicación la experiencia cerámica iniciada anteriormente en O Castro con un grupo de personalidades gallegas exiliadas en la República Argentina, cuyo trato va a ser uno de los acontecimientos fundamentales de su vida. Además de Luis Seoane, entre ellos se contaban Rafael Dieste, Lorenzo Varela, Nuñez Búa, Eduardo Blanco Amor o Arturo Cuadrado, de los cuales, "unos creían en la posibilidad del proyecto, otros no", pero todos ellos aplaudieron una posible actuación de propósitos restauradores de la memoria histórica de Galicia, ideas que enlazaban con los ensayos pioneros de producción cerámica llevados a cabo por Isaac en España.

Aunque Isaac conocía a varios de estos intelectuales con anterioridad a la Guerra Civil, el encuentro con Seoane fue especialmente importante, ya que supuso avivar una relación que durante muchos años había sido epistolar ${ }^{1}$, y que tendrá fecundas consecuencias en el futuro con la creación de la industria cerámica de Sargadelos y todas las experiencias de diseño a ella asociadas.

Por otra parte, Isaac llevó consigo hasta Argentina diferentes materiales de prensa, principalmente notas referentes a cuestiones sobre el expolio al que estaba siendo sometido el patrimonio gallego. $Y$, en especial, aquellas sobre un tema que entonces le tenía muy preocupado: el proyecto de traslado de la fachada de piedra del monasterio de Monfero a una nueva iglesia en construcción en uno de los barrios periféricos de A Coruña. Según relata el propio Isaac, con posterioridad a la guerra colaboró en la revista Galicia Emigrante publicada en la Argentina, en cuyas páginas se pueden encontrar las firmas de la mayoría de los intelectuales gallegos anteriormente mencionados ${ }^{2}$. Centrada en el ámbito cultural pero dotada también de una carga de reivindicación política, Galicia Emigrante, dirigida y diseñada por Luis Seoane, constituyó el órgano de difusión de noticias de Galicia entre la comunidad de exiliados y emigrantes gallegos ${ }^{3}$. En ella vio Díaz Pardo una magnífica ocasión para publicar las denuncias que en la prensa 
española suponían un riesgo y en la que, posiblemente, no tendrían cabida.

Aunque el primero de los artículos apareció firmado por Isaac Díaz Pardo ("Monfero", Galicia emigrante, $n^{\circ}$ 9, Febrero 1955, pp. 10-11), no ocurrió así con el segundo ("El despojo del patrimonio gallego", Galicia emigrante, $\mathrm{n}^{\circ} 18$, Febrero de 1956, pp. 1-2), sin duda debido a la dureza de las críticas y alusiones personales contenidas en sus líneas ${ }^{4}$. Ahora bien, en la entrevista que mantuvimos el pasado 19 de octubre de 2010 con el propio Isaac en su vivienda de Castro de Samoedo, recordó haber llevado hasta Argentina los materiales de prensa y notas referentes a los casos de expolio del patrimonio gallego denunciados en este y otros artículos, sin poder precisar si fue el único autor del mismo o si contó con la colaboración de Luis Seoane, siempre interesado en todo lo que afectara al arte y patrimonio de Galicia. En cualquier caso, creemos que es plausible afirmar la autoría de Díaz Pardo para ambos artículos puesto que no sólo comparten el mismo tema principal, criticando la intención de trasladar las piedras de la fachada del monasterio de Monfero hasta A Coruña, sino que los argumentos y estilo de redacción se repiten en los dos textos.

En el primero de los artículos, valiéndose del recurso a la narración en tercera persona protagonizada por un supuesto viajero, Díaz Pardo resume los principales valores históricos y artísticos reunidos en el monasterio de Monfero. Hay que destacar especialmente su acercamiento al monumento desde una perspectiva territorial, considerando los valores de su inserción en las tierras más inmediatas al conjunto monástico. Enlazando con un planteamiento ya adelantado por Ramón Otero Pedrayo en las líneas que había dedicado a este cenobio en su Guía de Galicia (1926), por este medio se asumen y reivindican como inseparables del monumento unos datos paisajísticos que aportan una de las principales argumentaciones para denunciar la irracionalidad de su traslado; de hecho, el propio Otero Pedrayo se hacía ya eco de esta posibilidad en la tercera edición de su Guía de Galicia (1954), al comentar lacónicamente que: "Se habla de trasladar a La Coruña, para la nueva parroquia de San Pedro de Mezonzo, la fachada de Monfero, de gran efecto ornamental. Quedaría triste la montaña".

Pese a su perfil histórico-artístico este primer artículo de Díaz Pardo se suma en sus últimas líneas a las mencionadas palabras de Otero Pedrayo al denunciar el proyecto de desmontar piedra a piedra la barroca fachada de Monfero para adosarla al nuevo templo de san Pedro de Mezonzo que por entonces estaba en construcción en A Coruña. Ya con anterioridad a la Guerra Civil, en agosto de 1929, el arzobispado de Santiago había autorizado la creación de esta nueva parroquia coruñesa, desgajada de la de santa Lucía. Como primer párroco fue nombrado José Toubes Pego (1881-1960), personaje muy vinculado al mundo de la prensa local ya que, como también se indica en el segundo artículo, había sido uno de los fundadores del periódico El Ideal Gallego. Aunque inicialmente esta parroquia tuviera como sede la capilla de santa Lucía, desde sus primeros tiempos era evidente la necesidad de construir un nuevo templo, de grandes proporciones de acuerdo con la ambiciosa visión de su párroco, dado que en su término se incluían todos los lugares habitados comprendidos entre las dos estaciones de ferrocarril, vieja y nueva, el puerto y la agrupación de industrias desarrolladas en aquella zona periférica de A Coruña, con rango de auténtico suburbio o barriada obrera de la capital desde finales del siglo XIX.

Desde la elaboración en 1943 del conocido como Plan Cort para la urbanización del extrarradio de A Coruña se reservó para la edificación de la nueva iglesia de Mezonzo una parcela situada en el lugar de Castiñeira de Abajo, en las inmediaciones de la fábrica de fósforos, la fábrica de cervezas Estrella Galicia y los talleres de la fundición Wonemburger. Los sucesivos planes de urbanización aprobados en 1945 y 1948 mantuvieron este mismo emplazamiento, con ligeras modificaciones para que ante la fachada de la nueva iglesia se abriera una amplia plaza y un paseo a enlazar con Cuatro Caminos y la futura avenida de Lavedra, todo ello con el fin de dar mayor protagonismo urbano al templo ${ }^{5}$. Por aquellos mismos años estaba en marcha una suscripción popular para adquirir los terrenos necesarios, si bien por sus escasos resultados a 
finales de 1944 el párroco Toubes consiguió que el gobierno de Franco, recogiendo una petición del arzobispo de Santiago Muñiz de Pablos, dictara un decreto declarando la urgencia de la obra y autorizando la expropiación forzosa de los terrenos necesarios ${ }^{6}$. Sin embargo, la resistencia a la venta de algunos propietarios de esos terrenos siguió dilatando en el tiempo el comienzo de las obras, que no pudieron iniciarse hasta el 15 de octubre del año 1952 ?

En el comentado contexto de dificultades para llevar adelante la ambiciosa basílica proyectada, el párroco Toubes consiguió entrevistarse con el general Franco, seguramente durante una de sus estancias veraniegas en Meirás. En dicha audiencia habría sido el propio Franco quien le sugirió la posibilidad de completar y realzar la construcción del templo trasladando hasta A Coruña la fachada de la iglesia monástica de Monfero ${ }^{8}$, con inclusión de uno de sus retablos ${ }^{9}$. Esta circunstancia, de la que se hizo eco la prensa de la época, no podía ser desconocida para Díaz Pardo, por lo que se comprende que, junto a la crítica a José Toubes, en su segundo artículo se introduzca una genérica mención a "las autoridades" que, secundadas por algunos periodis$\operatorname{tas}^{10}$ y técnicos ${ }^{11}$, habían promovido o estaban interviniendo en aquella polémica propuesta de traslado.

La idea de desmontar y reinstalar en la nueva ubicación la fachada de Monfero se justificó interesadamente entonces como la única salida posible ante la situación de ruina y abandono del monasterio. Declarado Monumento Nacional en 1931 gracias a su inclusión en la lista del macro decreto aprobado en los primeros meses de la II República (Decreto 03/06/1931), Monfero había sufrido sucesivos daños durante la Guerra de Independencia y el Trienio Liberal por la paralización de las obras que estaban en marcha y la primera expulsión de sus monjes. Lo peor llegó a partir de 1835, con motivo de la aplicación de las medidas de exclaustración y desamortización del gobierno de Mendizábal, ya que sus alhajas de plata fueron subastadas al peso, desapareció su archivo y biblioteca, y se produjo una sistemática expoliación de todo elemento que pudiera tener valor material alguno, desde las campanas a los herrajes, bisagras y cerraduras metálicas, cañerías, vidrieras, maderas de las puertas, ventanas y pisos, o las propias piedras de su fábrica, que redujeron algunas partes a un montón de escombros ${ }^{12}$. En la noche del 19 de febrero de 1879, la caída de un rayo terminó por desmoronar y echar por tierra la esquina norte de la fachada, incluyendo la torre de ese lado que ya no fue reconstruida, como se aprecia hoy $^{13}$.

Para avalar la solución técnica del traslado de la fachada de Monfero, el Servicio de Defensa del Patrimonio Artístico Nacional encargó en 1951 un informe a Francisco Pons-Sorolla Arnau, arquitecto-restaurador de la Primera Zona del Patrimonio Artístico Nacional. En dicho informe Pons-Sorolla manifestaba con claridad su preferencia por un traslado completo de toda la iglesia monástica, puesto que consideraba que no sería admisible "la adaptación de la fachada u otro elemento parcial a una basílica de moderno o distinto trazado", en evidente alusión a la opción de adosamiento parcial a la nueva parroquial coruñesa. Consecuente con esta idea de traslado completo, Pons opinaba que era necesario idear un "plan de conjunto" que permitiera salvar la mayor parte del histórico monasterio, ordenando de forma apropiada la plaza a crear en el nuevo emplazamiento, tanto en su superficie como en las edificaciones circundantes, ya que todo ello debía subordinarse a la adecuada valoración del monumento reubicado; aunque fuera sólo a título orientativo, el arquitecto se atrevía a estimar que el traslado y remontaje de la iglesia sobrepasaría los 10 millones de pesetas $^{14}$. Sin embargo, pese a lo afirmado en el informe de Pons-Sorolla, que consideraba irremediable la ruina del cenobio, la recuperación de Monfero no era en modo alguno inviable, puesto que son bien conocidos los casos de otros monasterios gallegos cuya reconstrucción sí estaba en marcha por aquellos mismos años, como Samos, Oseira y Sobrado, constituyendo este último el mejor término de comparación al haber dado inicio a su restauración en el año 1954, promovida por el arzobispo Quiroga Palacios.

La intervención de Pons-Sorolla permite abrir el enfoque sobre este proyecto de traslación de Monfero, puesto que en la misma época debe enmarcarse junto a otros polémicos casos que 
afectaron a la conservación del patrimonio gallego, y que el propio Díaz Pardo cita con sus menciones a la desaparición de las pinturas murales de Portomarín, finalmente restituidas en la iglesia reconstruida, y al saqueo de un supuesto tesoro localizado en el monasterio de Sobrado ${ }^{15}$. Precisamente el caso de Portomarín, donde en 1955 se proyectó el traslado de la iglesia de san Juan y otras piezas singulares del histórico enclave tan vinculado a la peregrinación jacobea, constituía un significativo precedente para el traslado de la fachada de Monfero, puesto que ambos se plantearon por las mismas fechas y con participación del mismo arquitecto-restaurador: Francisco Pons-Sorolla. Sin embargo, la justificación en aras del interés económico y progreso general que Portomarín compartía con otros templos afectados por la construcción de pantanos — san Pedro de la Nave por el pantano Ricobayo, san Esteban de Chouzán y san Juan da Cova por las obras del pantano de Os Peares-, haciendo preferible el traslado y remontaje de los monumentos en otro emplazamiento a su destrucción o desaparición, no podía bajo ningún concepto compararse a las circunstancias de Monfero.

Como bien apunta Díaz Pardo, a la ignorancia y desprecio por los valores del patrimonio gallego se unía en este caso la absurda renuncia a proyectar una iglesia nueva con materiales y lenguaje modernos, algo que irritaba especialmente a una persona como él, cuya ambición frustrada había sido realizar estudios de arquitectura. En cambio, la propuesta para reutilizar la fachada de Monfero fue la opción preferente durante años, concretada incluso en un proyecto que, desoyendo los razonamientos de PonsSorolla, mostraba cómo quedaría instalada en la nueva iglesia de Mezonzo, por supuesto, reconstruyendo en estilo la desaparecida torre norte $^{16}$. Esta solución sólo es parcialmente comprensible en el contexto de los citados precedentes en la traslación de otras iglesias gallegas, que en la misma ciudad de A Coruña tendrían continuidad con el posterior traslado de la igle- sia del convento de San Francisco. Pero también, y no en menor medida por su directa implicación en el asunto, cabe vincular esta actitud de disponer a voluntad de los bienes del patrimonio gallego con las actuaciones llevadas a cabo por el dictador Franco en su residencia veraniega de las Torres de Meirás. Formando parte de una concienzuda estrategia para apropiarse de la antigua morada de la escritora Emilia Pardo Bazán, siguiendo órdenes directas de Franco durante aquellos mismos años cuarenta y cincuenta se trasladaron e instalaron en los jardines de Meirás numerosas piedras armeras arrancadas de diferentes pazos y casas señoriales de toda Galicia y, lo que todavía es más grave, fuentes, portadas y partes de las fábricas de pazos de tanto valor artístico como los de Ximonde o Dodro; de hecho, con las piedras de la torre y balconada del pazo de Dodro se compuso la escalinata y balaustradas que salvan el desnivel entre la primera y segunda terraza de los jardines, como todavía se aprecia hoy en día. Por lo tanto, la genérica alusión a las "autoridades" que estaban apoyando el traslado de la fachada de Monfero escondía otra intencionada crítica a la figura de Franco, que ayuda a entender porqué este segundo artículo apareció publicado sin firma.

Las duras valoraciones que Díaz Pardo realiza sobre lo que califica de "injuria" y "traición al país" concluyen con un llamamiento a la responsabilidad para que, abandonando el proyectado traslado, no sólo se restaurara el monasterio de Monfero, sino que se contribuyera a su conservación futura en el paisaje que lo había visto surgir. Afortunadamente las dificultades técnicas y el elevado coste del traslado de las piedras de la fachada de Monfero se aliaron con la firme oposición de algunos intelectuales de prestigio, como Ángel del Castillo ${ }^{17}$, para conseguir que se abandonara aquella injustificable y absurda solución, y se abriera el camino, si bien con demasiado retraso, a la recuperación del monasterio actualmente en marcha. 
NOTAS

${ }^{1}$ X. Díaz, L. Muñoz, J. Rodríguez, Arte e industria: Isaac Diaz Pardo, COAG-Labirinto de Paixóns S.L., A Coruña, 2001, pp. 116-117.

2 "Tenía una relación epistolar con Seoane antes de ir para allá. El estaba ya con la revista Galicia Emigrante y yo colaboré en ella. Y entonces cuando llegué allí ya tenía relación con todos ellos". X. Díaz, L. Muñoz, J. Rodríguez, op. cit., p. 117.

${ }^{3}$ La revista Galicia Emigrante fue fundada por Luis Seoane en 1954, editándose hasta 1959, período en el que aparecieron sus 37 números. X. Lama, R. González Bello, "A liña editorializante da revista Galicia emigrante" en O exilio galego de 1936: política, sociedade, itinerarios (X.M. Núñez Seixas e P. Cagiao Vila eds.), Ediciós do Castro, Sada-A Coruña, 2006, pp. 323-343. Tuvo también una versión emitida por radio desde el año 1956, dedicada a charlas sobre temas gallegos, recogidas luego en el libro: L. Seoane, Comunicacións Mesturadas, Galaxia, Vigo, 1973.

${ }^{4}$ De hecho X. Lama y R. González Bello han destacado que, al margen de los editoriales redactados por Luis Seoane, las denuncias y contenidos más polémicos de la revista se localizan en los artículos firmados por Díaz Pardo, bien directamente o a través del seudónimo "S. Fernández Ollarnovo". X. Lama y R. González Bello, op.cit., p. 326.

${ }^{5}$ Sobre el origen y ordenación de este sector de Cuatro Caminos en los distintos planeamientos de la época véase J. González-Cebrián Tello, La ciudad a través de su plano. La Coruña, Ayuntamiento de La Coruña, 1984.

6 Herculano, "La iglesia parroquial de San Pedro de Mezonzo", La Voz de Galicia, 25 de enero de 1945 , p. 2.

7 Con proyecto definitvo del arquitecto Francisco Echenique, las obras fueron sufragadas primero con cargo a los presupuestos de la Direc- ción General de Regiones Devastadas, y cuando esta desapareció con los de la Dirección General de Arquitectura, dentro del Ministerio de la Vivienda. Datos consignados con motivo de la inauguración de la iglesia en "Ayer fue solemnemente inaugurado el nuevo templo de San Pedro de Mezonzo", La Voz de Galicia, 10 de septiembre de 1964, pp. 1 y 9.

${ }^{8}$ Un artículo publicado en 1946 así lo corrobora, esgrimiendo además el interés que para A Coruña supondría contar con un monumento de tal categoría, como si se tratara de una competición para ponerse a la altura de otros conjuntos históricos gallegos, pensando en Santiago de Compostela: "Hay diversas sugestiones magníficas en torno a la obra. Una tan admirable como la de S.E. el Caudillo, de trasladar a La Coruña un retablo y la decorativa fachada de un monasterio secular, hoy en ruinas, para adaptar ésta como pórtico a la presunta iglesia, corre el riesgo de perderse... ¡Con lo faltos que estamos aquí de suntuosas obras arquitectónicas de otros siglos!". Herculano, "Solución que urge. La iglesia parroquial de San Pedro de Mezonzo", La Voz de Galicia, 30 de agosto de 1946, p. 2.

9 Seguramente se trataba del retablo mayor, realizado en 1693 , que en la actualidad todavía se conserva, aunque desmontado y con pérdida de parte de sus piezas, en el coro alto de la iglesia monástica. J.L. López Sangil, Historia del Monasterio de Santa María de Monfero, Diputación Provincial de A Coruña, 1999, p. 116.

${ }^{10}$ Entre esos periodistas, las críticas de Díaz Pardo se centran en el autor de un libro titulado La traición como arte, obra efectivamente publicada en 1955 por el periodista gallego Felipe Fernández Armesto, corresponsal de La Vanguardia y La Voz de Galicia, que firmaba sus crónicas bajo el seudónimo "Augusto Assía".

${ }^{11}$ La alusión a un nieto del historiador Martínez Salazar que figura en el texto se refiere al ingeniero industrial coruñés Juan María Martínez-Barbeito, cuya empresa constructora se había adjudicado las obras de edificación del nuevo templo de Mezonzo.

12 Una pormenorizada relación de estos expolios, y los precedentes destrozos en las alhajas, muebles, vidrios y espejos producidos durante el Trienio Liberal, aparece recogida por J.L. López Sangil, op. cit., pp. 98-101. En cuanto a los frustrados intentos para recuperar el monasterio a finales del siglo XIX, incluyendo una efímera reinstalación de los monjes bernardos en 1882, se documenta en Fr. M ${ }^{a} D$. Yáñez Neira, "El último capítulo en la historia de Monfero", Abrente, no 10 y 11, 1978 y 1979, pp. 33 a 58 y 49 a 79.

${ }^{13}$ La "ignominiosa y degradante" destrucción de Monfero suscitó ya en su momento amargas palabras de Antonio de la Iglesia, dentro de una serie de artículos en los que proponía una decidida actuación para solucionar la ruina de los principales monasterios gallegos ("Estudios arqueológicos. Monasterio de Monfero", Galicia. Revista Universal de este Reino, año IV, $n^{\circ} 10,11$ y 12, 15 de mayo, 1 de junio y 15 de junio de 1863), comentado en M.R. Saurín de la Iglesia, Antonio, Francisco y Benigno de la Iglesia: una biografía intelectual, Instituto de Estudios Gallegos Padre Sarmiento, Santiago de Compostela, 2003; y J.A. Sánchez García, "Una década trágica para el patrimonio gallego. De la desamortización a las Comisiones de Monumentos (1835-1844)", Quintana, $n^{\circ}$ 3, 2004, pp. 123-151.

${ }^{14}$ Según la documentación reunida sobre las intervenciones de PonsSorolla en Galicia por B. Castro Fernández, Francisco Pons-Sorolla y Arnau, arquitecto-restaurador: sus intervenciones en Galicia (19451985), Tesis Doctoral, Universidade de Santiago de Compostela, 2006, vol. II, pp. 53-56.

${ }^{15}$ Aunque sólo es objeto de una escueta mención, la conversión del antiguo Hospital de los Reyes Católicos compostelano en Parador de Turismo aparece recogida en el segundo artículo a través de la inserción de un recorte de prensa que pregonaba la paradójica convivencia de "un edificio 
del siglo XV con las comodidades de un hotel del siglo XX". El propio Díaz Pardo criticará en un artículo posterior algunos de los nuevos e inadecuados usos introducidos con motivo de esta rehabilitación, como la cafetería americana o la bolera que funcionaron durante algunos años en su interior. I. Díaz Pardo, "El Hospital Real de Santiago de Compostela", Galicia emigrante, n 19, Marzo-Abril de 1956, pp. 12-13; en este mismo artículo se citan otras polémicas intervenciones en Santiago, como el derribo de la casa de Espinosa para construir la sede del Banco de España en Platerías, o la reforma de la galería del Palacio Arzobispal.

${ }^{16}$ De acuerdo con un plano publicado en el diario La Noche: V. de las Heras, "Evolución progresiva de Galicia. Entusiasta aportación del ingeniero industrial D. Juan María Martínez-Barbeito en el desarrollo del extenso programa de edificaciones en nuestra región", La Noche. Suplemento de la Construcción, 3 de marzo de 1953.

${ }^{17}$ En el año 1959 Ángel del Castillo pudo publicar un artículo celebrando con satisfacción el abandono del proyectado traslado de la fachada "para mal vestir con ella, con la mejor intención, no cabe duda, pero en ana- crónico conjunto, la iglesia que de cemento armado se viene construyendo para parroquial de San Pedro de Mezonzo...". A. del Castillo, "De nuestra riqueza monumental. Hay que conservar el antiguo monasterio de Monfero", La Voz de Galicia, 22 de julio de 1959, p. 6. Citado por A. Vigo Trasancos, "Ángel del Castillo (18861961). Una vida dedicada al estudio y a la defensa del patrimonio artístico de Galicia" en Inventario de la Riqueza Monumental y Artística de Galicia (A. del Castillo), Reed. Fundación Pedro Barrié de la Maza, A Coruña, 2008, t. I, pp. 81 y 139. 